\title{
TITEL
}

Jost Reinecke, Mark Stemmler, Maria Arnis, Nibad El-Kayed, Julia Meinert, Andreas

Pöge, Deborah Schepers, Zara Sünkel, Burcu Uysal, Susanne Wallner, Maren Weiss, Jochen Wittenberg

\section{Entstehung und Entwicklung von Kinder- und Jugenddelinquenz: Erste Ergebnisse einer Längsschnittstudie}

\section{A. Einleitung}

Jugendliche unterliegen in ihrer körperlichen und geistigen Entwicklung zum jungen Erwachsenen vielfach Prozessen, die sowohl Chancen als auch Risiken für den Lebensverlauf beinhalten. Die Entwicklung einer Kultur, in der Abweichungen von gesellschaftlich konformen Verhaltensweisen als normal empfunden werden, hängt mit Ablösungsprozessen von den primären Erziehungsinstanzen zusammen ${ }^{1}$. Um die Entstehungsbedingungen normabweichender Verhaltensweisen, die bis zu strafrechtlich relevanten Taten reichen können, sowie deren Beginn, Abbruch und Kontinuität zu untersuchen, sind Studien, die den Altersverlauf im Untersuchungsdesign berücksichtigen, notwendig. Im Vordergrund unserer Untersuchung steht der Zusammenhang zwischen der Entwicklung dieser Verhaltensweisen und der Verfestigung sozialer Ungleichheiten sowie sozialer Ausgrenzung. Es sollen im Verlauf unserer Untersuchung Faktoren identifiziert werden können, die „Ausstiegsprozesse“ aus devianten und delinquenten Verhaltensweisen begünstigen und den „Einstieg“ in normalbiografische Lebensverläufe fördern. Darüber hinaus stehen auch Faktoren im Vordergrund, die eine längerfristige, über das Jugendalter hinausgehende Persistenz abweichender Verhaltensweisen begünstigen $^{2}$.

Während statisch ausgerichtete Erklärungsansätze wie der von Gottfredson und Hirschi (1990) davon ausgehen, dass normabweichendes Verhalten auf stabilen Persönlichkeitseigenschaften und entsprechenden sozialen Dispositionen beruht, richtet sich eine dynamisch ausgerichtete Lebensverlaufsforschung sowohl auf die Untersuchung von Veränderungen individueller Einstellungen und individuellen Verhaltens als auch auf den Wandel sozialer Kontexte und ihrer Kontrollstrukturen (z.B. Familie, Schule, Peers, Polizei, Justiz). Eine dynamisch ausgerichtete Lebensverlaufsforschung ermög- 
licht die gleichzeitige Analyse interindividueller Gruppenvergleiche und intraindividueller Entwicklungsverläufe. Über Geschlecht und Migrationshintergrund beobachtete Unterschiede können dabei gleichzeitig mit den sich aus den Delinquenzverläufen zeigenden Differenzen untersucht werden. Die Methodologie und die damit verbundenen statistischen Modellierungsmöglichkeiten werden mittlerweile anwendungsorientiert diskutiert $^{3}$. Die hier vorgestellte Untersuchung knüpft an die kriminologische Lebensverlaufsforschung ${ }^{4}$ an und versucht, sowohl die Bedeutung formeller und informeller Kontrollinstanzen im Lebensverlauf als auch die situationsbezogene Analyse der Entstehung und Entwicklung von Devianz und Delinquenz zu erforschen. Die dafür notwendigen theoretischen Ansätze und Konzeptionen werden zugunsten der ersten empirischen Befunde an anderer Stelle vorgestellt $t^{5}$.

Nach einer kurzen Darstellung der polizeilich registrierten Kriminalität im Hellfeld (bezogen auf Bayern und NRW) werden die auf Basis der Selbstberichte ermittelten Jahresprävalenzen für den Dortmunder und Nürnberger Untersuchungskontext diskutiert. Hieran schließen sich Befunde zum Konsum von Alkohol und Drogen an. Zusammenhänge zwischen Delinquenz und Migrationshintergrund, die Bedeutung von Werten, Freizeitverhalten und Medienkonsum sowie die Relevanz der klassischen Kontrollinstanzen Schule, Familie und Peers stehen im weiteren Fokus der ersten Untersuchungsergebnisse. ${ }^{6}$

\section{B. Beschreibung von Erhebungsdesign und Stichprobe}

Die Gesamtstichprobe der Schülerbefragungen, die im Frühjahr 2012 schriftlich in den Klassenräumen durchgeführt wurden, besteht aus zwei Teilstichproben für die Erhebungsorte Dortmund und Nürnberg. Neben einem Vergleich der beiden Städte verfolgt die Studie ein sogenanntes Kohorten-Sequenz-Design. Dies ermöglicht sowohl den Vergleich zweier Alterskohorten (beginnend mit Kindern in der 5. Jahrgangsstufe und Jugendlichen der 9. Klassen), wie auch die Analyse zeitlicher Entwicklungen. Hierfür wurden die Schüler, die 2012 an der Befragung teilnahmen, auch in diesem Jahr erneut befragt. Eine Fortsetzung der Befragungen in den kommenden Jahren bietet die Chance, sowohl den Kohortenvergleich zu intensivieren (so erreicht die jüngere Kohorte 2016 ebenfalls die Jahrgangsstufe 9), wie auch individuelle Verläufe der Delinquenzbelastung von der Kindheit bis zum jungen Erwachsenenalter detailliert zu beobachten.

Während in Dortmund Schulen aller Schulformen beteiligt waren, besteht das Nürnberger Sample nur aus Mittelschulen (in Bayern werden Hauptschulen als Mittelschulen bezeichnet). Für Nürnberg liegen 529 Fragebögen der 5. Jahrgangsstufe und 494 der 9. Jahrgangsstufe vor. In Dortmund konnten 807 Bögen in der 5. und 927 in der 9. Jahr-

3 Vgl. Reinecke 2012.

4 Vgl. Boers et al. MschrKrim 92 (2009), 67.

5 Reinecke et al. 2013.

6 Die hier dargestellte Studie ist Teil des Projekts A2 des Sonderforschungsbereichs „Von Heterogenitäten zu Ungleichheiten“ (SFB 882), der von der DFG an der Universität Bielefeld gefördert wird (vgl. Diewald/Faist BJS 21(2011), 91). 
gangsstufe gewonnen werden. Insgesamt beteiligten sich 37 Schulen mit 192 Klassen an der Befragung.

\section{Kinder-und Jugendkriminalität im Hell-und Dunkelfeld}

Ein kurzer Blick auf das Hellfeld der polizeilich registrierten Kriminalität von Kindern und Jugendlichen verdeutlicht, dass es in den letzten Jahren zu einem Rückgang der Registrierungen gekommen ist: Sowohl für das gesamte Bundesgebiet, wie auch die hier näher betrachteten Bundesländer Bayern und Nordrhein-Westfalen (NRW) gehen sowohl für Kinder und Jugendliche als auch für Heranwachsende die Tatverdächtigenbelastungsziffern (TVBZ) zurück und liegen zum Teil deutlich unter denen, die noch im Jahr $2000 \mathrm{zu}$ verzeichnen waren (vgl. Schaubild 1). Eine entsprechende Entwicklung ist auch für die absolute Anzahl registrierter Delikte und Tatverdächtiger zu beobachten. ${ }^{7}$ Im regionalen Vergleich zeigt sich, dass die registrierte Kriminalität in NRW insgesamt über der von Bayern und dem Bundesdurchschnitt liegt. Mit Blick auf die im Folgenden dargestellten Dunkelfelddaten kann darüber hinaus festgestellt werden, dass die Stadt Dortmund deutlich höhere Belastungsziffern aufweist als der Landesdurchschnitt, und dass für die Stadt Nürnberg die Werte über denen von Mittelfranken und Bayern liegen $^{8}$; so liegt die TVBZ für Dortmund bei den 8-14-Jährigen im Jahr 2012 bei 1.919 (2011: 2.493), bei den Jugendlichen 2012 bei 11.448 (2011: 12.165) und bei den Heranwachsenden bei 15.213 (2011: 15.074) . Wie in zahlreichen anderen Analysen lässt sich auch hier zeigen, dass männliche Tatverdächtige die Mehrheit der registrierten Kinder und Jugendlichen ausmachen.

7 Betrachtet man nur registrierte Gewaltdelikte, so ist erst seit etwa 2007 ein Rückgang der Kennziffern zu erkennen. Vgl. PKS-Zeitreihe, Tabellen 20, 40, 50 (www.bka.de).

8 Vgl. Polizeipräsidium Mittelfranken, Kriminalstatistik 2012, Nürnberg.

9 Vgl. Landeskriminalamt NRW, 2013, persönliche Mitteilung der Kriminologischen Forschungsstelle.

NK 25. Jg. 3/2013 
Schaubild 1: Tatverdächtigenbelastungsziffern (TVBZ) in Bund, Bayern und NRW differenziert nach Alter, 2000-2012

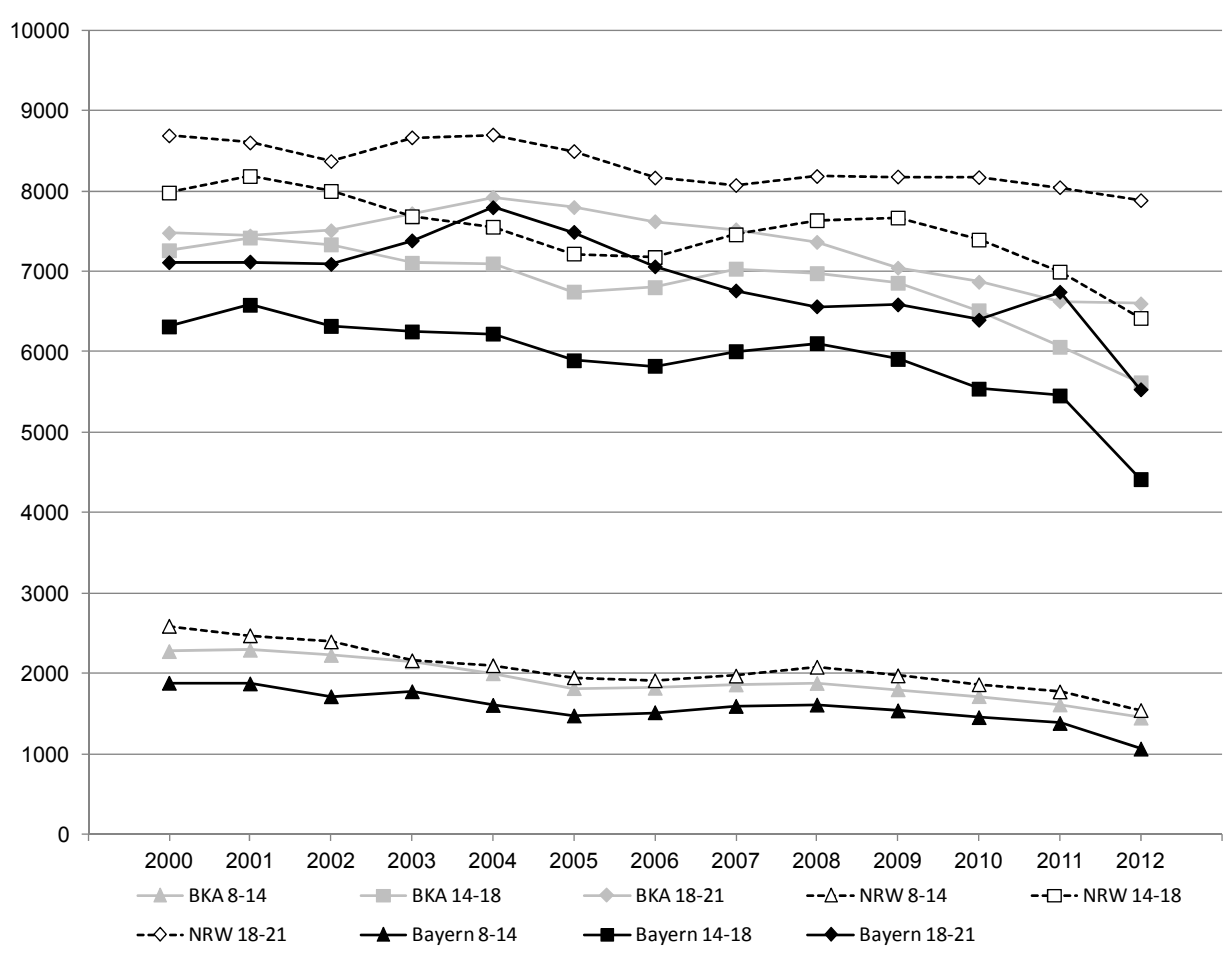

Quellen: Polizeiliche Kriminalstatistik des Bundeskriminalamtes (Tabelle T61), Jahrbücher der Landeskriminalämter Bayerns und Nordrhein-Westfalens

Die altersspezifische Deliktstruktur soll hier am Beispiel der Polizeilichen Kriminalstatistik (PKS) für Bayern veranschaulicht werden: Wenn Kinder polizeilich registriert werden, erfolgt dies überwiegend wegen leichter Diebstahlsdelikte, insbesondere wegen Ladendiebstählen. Mit deutlichem Abstand folgen Sachbeschädigungen und Körperverletzungen. Im Zeitverlauf nimmt dabei die Bedeutung der Ladendiebstähle ab und ist für einen erheblichen Teil des Gesamtrückgangs verantwortlich. Bei Jugendlichen (14-18 Jahre) kommen Delikte im Zusammenhang mit Rauschgiften (nach BtMG) als weitere Deliktgruppe hinzu. Im Vergleich der letzten zehn Jahre nimmt jedoch die Bedeutung von Rauschgiftdelikten ebenso wie die des Ladendiebstahls als Registrierungsgrund ab. Beide Delikte hängen in ganz erheblichem Umfang von der Anzeigepraxis bzw. Kontrollintensität ab. Der Rückgang erscheint jedoch so erheblich, dass er wahrscheinlich nicht nur auf die Registrierungspraxis zurückzuführen ist ${ }^{10}$.

10 Vgl. Polizeipräsidium Mittelfranken, Kriminalstatistik 2012, Nürnberg. 
Ausgehend von den oben für Dortmund berichteten Registrierungsraten lässt sich der Anteil polizeilich registrierter Kinder auf etwa $2 \%$ und für Jugendliche auf $11 \%$ beziffern. Dem kann man vergleichend den mit $8 \%$ ähnlich hoch geschätzten Anteil der durchschnittlich etwa 15 Jahre alten Schülerschaft der 9. Klassen in Dortmund gegenüberstellen, der in unserer Dunkelfeldbefragung für zumindest ein Delikt angegeben hat, dass eine Registrierung durch die Polizei in den letzten zwölf Monaten erfolgte. Auch in den Befragungsdaten finden sich deutlich höhere Werte für die männlichen Befragten.

Während die Stärken der PKS-Daten in der detaillierten regionalen Untergliederung sowie der Verfügbarkeit von Zeitreihen für Trendanalysen bestehen, erlaubt die weitere Auswertung der Befragungen in Dortmund und Nürnberg einen genaueren Blick auch auf das Dunkelfeld der Delinquenz von Kindern und Jugendlichen. Im Fragebogen der Jahrgangsstufe 5 wurde den befragten Kindern eine Liste von 14 Delikten vorgelegt, während in den 9. Klassen eine auf 24 Delikte erweiterte Version zum Einsatz kam, die auch schwerere und altersspezifisch erst hier sinnvoll erfragbare Delikte (z.B. Fahren ohne Fahrerlaubnis) umfasste. Tabelle 1 gibt eine Übersicht über die Jahresprävalenzen der genannten Delikte. Die Dortmunder Stichprobe wurde hierfür nach Schulform gewichtet. In Nürnberg wurde die Befragung ausschließlich an Mittelschulen durchgeführt.

In der 5. Jahrgangsstufe wird in Dortmund nur in seltenen Fällen von zurückliegenden Delikten berichtet. Meist geben nur 1 bis $2 \%$ der befragten Kinder an, die abgefragten Delikte begangen zu haben. Vergleichsweise häufig werden in erster Linie Sachbeschädigungsdelikte genannt, aber auch hier liegen die Anteilswerte unter $5 \%$. An Nürnberger Mittelschulen der gleichen Altersstufe sind die Werte höher als in Dortmund, was jedoch im Wesentlichen auf die andere Zusammensetzung der Stichprobe und kaum auf stadtspezifische Unterschiede zurückzuführen ist. Die im 9. Jahrgang befragten Jugendlichen berichten nahezu ausnahmslos für alle Delikte höhere Raten, wobei erst die Fortsetzung der Studie in den folgenden Jahren zeigen wird, in welcher Altersstufe die höchste Belastung erreicht wird bzw. wurde. Im Deliktspektrum der Jugendlichen spielen neben den Sachbeschädigungen insbesondere Diebstahlsdelikte eine Rolle. Aber auch Körperverletzungen und (devianter) Drogenkonsum werden in nennenswertem Umfang berichtet (letzteres berichten knapp $13 \%$ bzw. $15 \%$ der Befragten). Ein Vergleich der Nürnberger Mittelschüler mit den Jugendlichen, die in Dortmund eine Hauptschule besuchen, führt zu recht ähnlichen Ergebnissen. Schwere Delikte und insbesondere schwere Gewaltdelikte sind auch im 9. Jahrgang die klare Ausnahme. 
Tabelle 1: Jahresprävalenzen verschiedener Delikte nach Stadt und Jahrgang

\begin{tabular}{lllll}
\hline & DO 5 & NÜ 5 & DO 9 & NÜ 9 \\
\hline Graffiti/Tags & 2,5 & 5,8 & 7,6 & 5,1 \\
Scratching & 3,8 & 9,3 & 7,9 & 7,8 \\
Sachbeschädigung (Fenster u.ä.) & 2,0 & 4,0 & 4,3 & 6,1 \\
Sachbeschädigung (Zaun u.ä.) & 2,9 & 3,8 & 6,8 & 6,2 \\
Zündeln & 1,5 & 3,5 & 2,4 & 3,7 \\
\hline Automatenaufbruch & 3,5 & 7,9 & 7,7 & 10,0 \\
Einbruch & 2,0 & 4,1 & 9,8 & 8,2 \\
Diebstahl von Mitschülern & 2,5 & 5,2 & 5,7 & 6,2 \\
Diebstahl von einer Baustelle & 1,2 & 2,5 & 4,4 & 2,9 \\
Diebstahl in einer Gaststätte & - & - & 2,7 & 1,6 \\
Diebstahl in einem Kiosk & 1,7 & 3,9 & 5,3 & 5,7 \\
Diebstahl in einem Kaufhaus & 1,0 & 4,1 & 7,1 & 10,4 \\
Fahrraddiebstahl & 1,0 & 2,7 & 4,4 & 8,5 \\
Hehlerei & 1,2 & 1,7 & 4,3 & 4,1 \\
\hline Fahrzeugdiebstahl & - & - & 1,3 & 1,4 \\
\hline Fahren ohne Führerschein & - & - & 19,0 & 21,4 \\
\hline Belästigung & - & - & 6,8 & 8,6 \\
\hline sexuelle Belästigung & - & - & 1,8 & 3,3 \\
\hline Körperverletzung ohne Waffe & - & - & 8,8 & 14,2 \\
Raub & 1,1 & 1,5 & 1,6 & 5,5 \\
Gewaltandrohung mit Waffe & - & - & 2,7 & 3,5 \\
\hline Körperverletzung mit Waffe & - & - & 1,4 & 2,9 \\
\hline Drogenkonsum & - & - & 12,9 & 14,8 \\
Drogenverkauf & - & - & 2,1 & 2,7 \\
\hline Anmerkwngen Gewchte Data & & & & \\
\hline
\end{tabular}

Anmerkungen: Gewichtete Daten. DO: Dortmund. NÜ: Nürnberg. 5: 5. Jahrgangsstufe. 9: 9. Jahrgangsstufe. -: nicht erhoben. Angaben in \%.

Für weitere Auswertungen werden statt der in Tabelle 1 dargestellten Einzeldelikte gruppierende Deliktbereiche verwendet. Hierfür wird zwischen Sachbeschädigungen (ohne Zündeln), Eigentumsdelikten und Gewaltdelikten unterschieden. Zusätzlich wird ein Index zur Gesamtkriminalität verwendet, der jedoch die Belästigungsdelikte, das Fahren ohne Fahrerlaubnis, den Drogenkonsum und das Zündeln unberücksichtigt lässt (vgl. Tabelle 2). Es wird deutlich, dass Eigentumsdelikte in beiden Alterskohorten die höchsten Prävalenzraten aufweisen. Mehr als jeder vierte Befragte in der Jahrgangsstufe 9 berichtet zumindest einen Diebstahl im vergangenen Jahr. Sachbeschädigungsdelikte, die in der Klasse 5 fast ebenso oft begangen werden wie Eigentumsdelikte, steigen zwar in der 9. Klasse an, haben aber dort einen deutlich niedrigeren Wert als die Eigentums- 
delikte. Während der Anteil an Gewalttätern in der jüngeren Kohorte unter $2 \%$ liegt, ist mehr als ein Zehntel der älteren Schüler zur Gruppe der Gewalttäter zu zählen - wobei leichte Gewalttaten überwiegen.

Tabelle 2: Jahresprävalenzen für gruppierte Delikte nach Stadt und Jahrgang

\begin{tabular}{lllll}
\hline & DO 5 & NÜ 5 & DO 9 & NÜ 9 \\
\hline Eigentumsdelikte & 7,9 & 16,2 & 26,1 & 30,8 \\
Sachbeschädigungsdelikte & 6,8 & 13,4 & 17,0 & 16,8 \\
\hline Gewaltdelikte & 1,1 & 1,5 & 11,3 & 18,6 \\
\hline Gesamt & 11,7 & 20,6 & 33,8 & 40,8 \\
\hline
\end{tabular}

Anmerkungen: Gewichtete Daten. DO: Dortmund. NÜ: Nürnberg. 5: 5. Jahrgangsstufe. 9: 9. Jahrgangsstufe. Angaben in \%.

Bereits die Gesamtprävalenz, bezogen auf das zurückliegende Jahr, gibt einen klaren Eindruck davon, dass insbesondere im Jugendalter delinquentes Verhalten in gewissem Umfang und mit mehrheitlich geringer Intensität keine Seltenheit darstellt: Etwa ein Drittel der älteren Befragtenkohorte in Dortmund und etwa $40 \%$ der Nürnberger Mittelschüler dieser Altersgruppe berichten mindestens eines der aufgeführten Delikte (Ubiquität). Aufgrund des Erhebungsdesigns ist nur für Dortmund eine schulformspezifische Auswertung möglich. Insgesamt zeigt sich, dass die Hauptschulen etwas stärker belastet sind als die anderen Schulformen. Die Gesamtschulen ähneln, nach Deliktsbereich und Jahrgangsstufe leicht variierend, mehr den Realschulen oder den Gymnasien. Die Unterschiede zwischen befragten Jungen und Mädchen sind in der Regel weitaus größer als die Schulformunterschiede. In der Klasse 5 berichten in Dortmund etwa $17 \%$ der Schüler zumindest ein Delikt, aber nur $6 \%$ der Schülerinnen (in Nürnberg sind es $26 \%$ bzw. $16 \%$ ). In der Klasse 9 bestätigt sich das Bild auf einem insgesamt gestiegenen Niveau. In Dortmund liegt der Täteranteil bei den männlichen Befragten bei knapp $40 \%$ gegenüber etwa $28 \%$ bei den weiblichen Jugendlichen (in Nürnberg sind es knapp $50 \%$ gegenüber etwa $32 \%$ ).

In beiden Städten und Alterskohorten ist eine kleine Gruppe mehrheitlich männlicher Befragter zu finden, die eine größere Zahl verschiedener Delikte berichtet. So begehen 2 bis $3 \%$ der etwa elfjährigen Schüler des jüngeren Jahrgangs fünf oder mehr verschiedene Delikte. Im 9. Jahrgang trifft dies sogar auf $7 \%$ (in Dortmund) bis $9 \%$ (in Nürnberg) der Befragten zu. Schaut man allein auf die Häufigkeit berichteter Gewalttaten, so kann man in beiden Städten für die 15-Jährigen jeweils eine Gruppe identifizieren, die durch intensivere Gewalttätigkeit (fünf und mehr Taten) auffällt. Der geschätzte Anteil dieser überwiegend männlichen Gewalttäter liegt bei etwa $4 \%$ in der Dortmunder Stichprobe und bei knapp $6 \%$ der Nürnberger Mittelschüler. Solche vielfältige bzw. intensive Tatbegehung charakterisiert einen kleinen, aber durchaus problematischen Teil der Jugendlichen, der für einen deutlich überproportionalen Anteil der gesamten Jugenddelinquenz verantwortlich ist. 
Insgesamt bestätigen diese Ergebnisse die Befunde anderer Studien zum Dunkelfeld der Jugendkriminalität. Im Vergleich zu den zahlreichen Dunkelfeldbefragungen, die das Kriminologische Forschungsinstitut Niedersachsen (KFN) seit Ende der 1990er-Jahre für Kinder und Jugendliche unterschiedlicher Großstädte und Bundesländer durchführte, fügen sich die Befunde aus Nürnberg und Dortmund in eine leicht sinkende Tendenz der Kriminalitätsbelastung im Zeitverlauf ein. Ein Großteil der Delikte wird heute seltener berichtet als vor etwa zehn Jahren. Auch die für Dortmund und Nürnberg berichteten Anteile an Gewalttätern bzw. intensiven Gewalttätern liegen auf ähnlichem Niveau wie in anderen Studien oder darunter ${ }^{11}$. Die in Dortmund im Jahr 2005 vom KFN durchgeführte Befragung in der 9. Klasse führte für Sachbeschädigungs- und Diebstahlsdelikte zu vergleichbaren Resultaten. Während Ladendiebstähle in der KFN-Studie deutlich häufiger berichtet wurden, ist in der Befragung 2012 der Anteil der berichteten Einbrüche erhöht ${ }^{12}$. Gewaltdelikte, die in der KFN-Studie für 2005 in Dortmund den höchsten Wert aller verglichenen Städte aufwiesen, liegen in der 9. Klasse der Studie 2012 auf erkennbar niedrigerem Niveau ${ }^{13}$. Auch die Ergebnisse der Längsschnittstudie „Kriminalität in der modernen Stadt", die seit 2000 für Münster und seit 2002 für Duisburg vergleichbare Daten erhebt, führen mit Ausnahme der Einbruchsdelikte zu höheren Werten, als in Dortmund für Sachbeschädigungen, Eigentums- und Gewaltdelikte zu verzeichnen sind.

\section{Alkobol- und Drogenkonsum}

Das Jugendalter stellt einen Lebensabschnitt dar, in dem erste Erfahrungen mit dem Konsum von Alkohol und Drogen gemacht werden. Befunde von Querschnitt- sowie Längsschnittstudien zum Verlauf des Alkohol- und Drogenkonsums über die Lebensspanne hinweg belegen, dass der Konsum im Durchschnitt während der Jugendphase besonders zunimmt und am häufigsten zwischen dem 18. und 25. Lebensjahr, also in der Übergangsphase zum Erwachsenenalter, auftritt ${ }^{14}$. Entsprechend den neuesten Daten der ESPAD-Studie 2011 (European school survey on alcohol and other drugs) konsumieren Jugendliche im europäischen Vergleich in Deutschland überdurchschnittlich häufig Alkohol (73\%). Europaweit betrachtet beginnen Jugendliche mit dem Alkoholkonsum durchschnittlich im Alter von etwa 13 Jahren.

Der Alkoholkonsum der Jugendlichen in unserer Befragung unterscheidet sich erwartungsgemäß eindeutig zwischen den Jahrgangsstufen. Rund $4 \%$ der Jugendlichen in der 5. Jahrgangsstufe haben bereits erste Erfahrungen mit Alkohol gemacht, dabei häufiger Jugendliche männlichen Geschlechts $(6 \%$ vs. $3 \%)$ und Jugendliche mit Migrationshintergrund (5\% vs. $1 \%$ ). Knapp $36 \%$ der Jugendlichen in der 9. Jahrgangsstufe geben an, dass sie schon einmal richtig betrunken waren. Hier trinken Jugendliche ohne

11 Vgl. Baier et al. 2006b, 135 ff.; 2009, 64, 95 ff.; 2010a, 180 f.; 2010b, 58 ff.; Baier/Pfeiffer 2011, $93 \mathrm{ff}$.; Baier/Rabold 2012, $77 \mathrm{ff}$.

12 Vgl. für die Jahresprävalenzen: Wittenberg et al. 2011, 129; Boers et al. NK 2/2010, 58; vgl. für Deliktverläufe im Längsschnitt Boers et al. EJC 7(2010),499.

13 Vgl. Baier et al. 2006b, $135 \mathrm{ff}$.

14 Vgl. BZgA 2012. 
Migrationshintergrund häufiger Alkohol (38\% vs. $33 \%$ ). Bei Erstkonsum sind die Jugendlichen im Durchschnitt 14 Jahre alt. Von den $36 \%$ der Jugendlichen mit Alkoholkonsumerfahrungen betrinken sich rund $20 \%$ mehrmals im Monat und knapp $5 \%$ mehrmals die Woche.

Auch die Bewertung des Trinkens fällt in den Jahrgangsstufen sehr unterschiedlich aus: Fünftklässlerinnen und Fünftklässler finden es bedeutend schlimmer, wenn sich Jugendliche in ihrem Alter betrinken. Jungen beider Altersgruppen finden es weniger schlimm, Alkohol zu trinken. Darüber hinaus lässt sich in der 9. Jahrgangsstufe ein Unterschied bei Jugendlichen ohne Migrationshintergrund feststellen: Sie schätzen das Betrunkensein als weniger schlimm ein. ${ }^{15}$ Damit spiegelt die Bewertung des Alkoholkonsums Gleichaltriger die Befunde des eigenen Konsums wider.

In der 9. Jahrgangsstufe wurden die Jugendlichen zusätzlich zu ihrem Drogenkonsum befragt. Etwa $17 \%$ haben bereits mindestens einmal in ihrem Leben illegale Drogen genommen. Auch hier sind die Jugendlichen bei Erstgebrauch im Durchschnitt 14 Jahre alt. Vergleichbar mit den Ergebnissen zum Alkoholkonsum haben Jugendliche männlichen Geschlechts häufiger Drogen konsumiert.

In beiden Jahrgangsstufen wurden die Jugendlichen gebeten, den Drogenkonsum ihrer Freunde einzuschätzen. Dabei nehmen $94 \%$ der Fünftklässlerinnen und Fünftklässler und $61 \%$ der Neuntklässlerinnen und Neuntklässler an, dass ihre Freunde keine Drogen konsumieren. Knapp $13 \%$ der Jugendlichen in der 9. Jahrgangsstufe geben an, dass ihre Freunde regelmäßig Drogen nehmen. Jungen bewegen sich häufiger in Freundeskreisen, in denen Drogen konsumiert werden. Die Ergebnisse zur Bewertung von Drogenkonsum sind vergleichbar mit denen zum Alkoholkonsum und spiegeln ebenfalls die Befunde zum eigenen Konsum wider. Neuntklässlerinnen und Neuntklässler bewerten den Drogenkonsum als weniger schlimm als Fünftklässlerinnen und Fünftklässler, während Jugendliche weiblichen Geschlechts das Konsumieren von Drogen für moralisch bedenklicher halten als Jugendliche männlichen Geschlechts.

Ausgehend davon, dass Alkohol- bzw. Drogenkonsum im Jugendalter eine Normverletzung ausdrückt, stellt sich im Rahmen unserer Untersuchung auch die Frage, inwiefern weitere Normverletzungen (Delikte) von Jugendlichen begangen werden, die schon Erfahrungen mit Alkohol- bzw. Drogenkonsum gemacht haben. Der Vergleich von Tätern und Nichttätern hinsichtlich des Konsums zeigt deutliche Unterschiede: Betrachtet man lediglich Täterinnen und Täter, so haben knapp $20 \%$ der Befragten der 5 . Klassen bereits erste Erfahrungen mit Alkohol gemacht. Bei Fünftklässlerinnen und Fünftklässlern, die dagegen keines der Delikte begangen haben, verringert sich der Anteil der Befragten, die Alkohol konsumieren, auf nur etwas über $1 \%$. Ähnlich sieht die Verteilung in der 9 . Klasse aus. Hier waren erst $20 \%$ der männlichen und weiblichen Nichttäter bereits betrunken, im Gegensatz zu $62 \%$ derjenigen, die im vergangenen Jahr de-

15 Knapp $42 \%$ der Jugendlichen ohne Migrationshintergrund bewerten das Betrunkensein als nicht schlimm bis überhaupt nicht schlimm, bei Befragten mit Migrationshintergrund sind es $34 \%$. Auf der anderen Seite geben $35 \%$ der Neuntklässlerinnen und Neuntklässler ohne Migrationshintergrund an, dass sie es schlimm bis sehr schlimm finden sich zu betrinken, der Anteil erhöht sich bei Befragten mit Migrationshintergrund auf $44 \%$.

NK 25. Jg. 3/2013 
linquentes Verhalten gezeigt haben. Die Prävalenzen zu Drogenkonsum und Delinquenz weisen ebenfalls in die gleiche Richtung: $37 \%$ der Täter haben bereits Drogen konsumiert, im Vergleich zu $5 \%$ der befragten Jugendlichen, die im vergangenen Jahr kein delinquentes Verhalten gezeigt haben.

\section{E. Migrationshintergrund}

Ein stark diskutierter Teilaspekt der kriminalsoziologischen Jugendforschung ist „Migration und Kriminalität". Jedoch kommen bisherige Studien zu heterogenen Ergebnissen in Bezug auf die Kriminalitätsraten von Jugendlichen mit Migrationshintergrund ${ }^{16}$. Im Folgenden wird der Migrationshintergrund einer Person analog zur Definition des Statistischen Bundesamtes bestimmt. ${ }^{17}$

In der 5. Klasse haben $11 \%$ der Kinder ohne Migrationshintergrund schon einmal ein strafrechtlich relevantes Delikt begangen, während der Anteil bei den Kindern mit Migrationshintergrund $17 \%$ beträgt. Differenziert nach Deliktarten, findet man eine ähnliche Relation. $6 \%$ der Fünftklässlerinnen und Fünftklässler ohne Migrationshintergrund bzw. $11 \%$ der Schülerinnen und Schüler mit Migrationshintergrund haben schon einmal ein Sachbeschädigungsdelikt begangen. Bezogen auf Eigentumsdelikte, haben $8 \%$ der Kinder ohne Migrationshintergrund und $13 \%$ der Kinder mit Migrationshintergrund schon einmal ein solches Delikt begangen. ${ }^{18}$ Zusammenfassend kann jedoch nicht von einem deutlichen Unterschied gesprochen werden. In der 9. Klasse ist kein Unterschied mehr erkennbar, weder im Gesamtindex noch bei Eigentums- und Sachbeschädigungsdelikten. Lediglich bei Gewaltdelikten ist die Differenz zwischen beiden Gruppen größer, bleibt jedoch insgesamt gering: 11 \% der Schüler ohne Migrationshintergrund haben schon einmal ein Gewaltdelikt begangen gegenüber $16 \%$ der Schüler mit Migrationshintergrund.

Man könnte argumentieren, dass diese von anderen Studien abweichenden Ergebnisse durch die relativ weite Definition des Migrationshintergrundes begründet sind. Dies bestätigt sich jedoch nicht, wenn man die Täterraten nach Staatsbürgerschaft strukturiert - dem Merkmal, das auch die Polizeiliche Kriminalstatistik verwendet. In den meisten Deliktgruppen verringern sich die Differenzen noch weiter oder bleiben gleich. Allerdings ergibt sich bei den Eigentumsdelikten in der 9. Klasse ein anderes Ergebnis: Schüler mit deutscher Staatsbürgerschaft haben eine um $9 \%$ höhere Täterrate (30\%) als Schüler mit einer anderen Staatsbürgerschaft (21\%). Im Gesamttäterindex für die 9 . Klasse findet sich dieses Verhältnis wieder: $38 \%$ der Schüler mit deutscher Staatsbürgerschaft haben

16 Vgl. Strobmeier EuU 9-10/2007, 796; Baier et al. 2006a; Boers et al. MschrKrim 89 (2006), 63.

17 Vgl. Statistisches Bundesamt 2012, 5 f. Damit haben Personen einen Migrationshintergrund, die nach 1949 auf das heutige Gebiet der Bundesrepublik Deutschland zugewandert sind sowie alle in Deutschland geborenen Personen ohne deutsche Staatsangehörigkeit und alle in Deutschland mit deutscher Staatsangehörigkeit Geborenen mit zumindest einem zugewanderten oder ohne deutschen Pass in Deutschland geborenen Elternteil.

18 Gewaltdelikte werden in dieser Altersgruppe nicht betrachtet, da kaum ein Kind in der 5. Klasse schon einmal ein Gewaltdelikt begangen hat. 
schon einmal ein strafrechtlich relevantes Delikt begangen, während dies nur für $31 \%$ der Schüler mit einer nicht-deutschen Staatsbürgerschaft zutrifft. Bei den Gewaltdelikten reduziert sich die Differenz zwischen den beiden Gruppen hingegen auf $2 \%$ (mit deutscher Staatsbürgerschaft: $16 \%$, ohne deutsche Staatsbürgerschaft: $14 \%$ ). Insgesamt bestätigen die Befunde die Ergebnisse von Boers et al. (2006), dass es weder in Bezug auf Gewaltdelikte noch in Bezug auf andere Deliktarten deutliche Unterschiede zwischen Jugendlichen mit und ohne Migrationshintergrund gibt.

\section{F. Werte}

Menschlichen Werten wird gemeinhin Handlungsrelevanz in Bezug auf delinquentes Verhalten zugesprochen. Besonders konservativ-religiös-traditionelle Werte auf der einen und materialistisch-hedonistische auf der anderen Seite spielen dabei eine Rolle. Werte können dabei direkt ${ }^{19}$ oder über durch sie konstituierte Gruppen wirken ${ }^{20}$. In unserer Untersuchung der 9. Jahrgangsstufe wird zur Wertemessung eine verkürzte, fünfstufige Sinus-Skala verwendet ${ }^{21}$, die die nach Inglehart (1979) oder Klages (1984) soziologisch relevanten Dimensionen „Tradition“ und „Hedonismus“ enthält.

Etwas mehr als zwei Drittel (67\%) der befragten Jugendlichen stimmen der Wertedimension „Tradition“, etwas weniger als die Hälfte (46\%) der Dimension „Hedonismus" zu. ${ }^{22}$ Die grundlegende Werteorientierung eines Menschen wird allerdings nicht allein durch Zustimmung oder Ablehnung einer einzigen Wertedimension geprägt, sondern durch die Kombination verschiedener Werte. In Anlehnung an Klages und Gensicke (2006) können mit Hilfe eines einfachen Vier-Felder-Schemas der beiden Wertedimensionen vier verschiedene Wertetypen gebildet werden. 1) Traditionelle (34\%), 2) Hedonisten (13\%), 3) Wertesynthetiker (33\%) und 4) Ablehnende (11\%). ${ }^{23}$ Etwas überraschend ist die Erkenntnis, dass das Profil der „Traditionellen“ am weitesten und das der „Hedonisten“ am wenigsten weit verbreitet ist, geht man doch üblicherweise von Hedonismus als einer gerade in der Jugendphase prägenden Wertedimension aus. In unserer Untersuchung tritt Hedonismus zudem häufig in Kombination mit den klassischerweise diametral angenommenen traditionellen Werten in Erscheinung. Ein Drittel der Jugend-

19 Vgl. Hermann 2003; Reinecke 2007.

20 Vgl. Heitmeyer et al., 1995; Boers/Pöge 2002; Pöge 2007.

21 Vgl. Flaig et al. 1994; Heitmeyer et al. 1995.

22 Bei den traditionellen Werten sind dabei in Bezug auf Geschlecht und Migrationshintergrund deutliche Unterschiede zu verzeichnen. Vermutlich durch das sehr konservative Geschlechterbild, das in den Einzelfragen zum Ausdruck kommt, lässt sich die Differenz von etwa 10 Prozentpunkten zwischen Jungen (71 \% Zustimmung) und Mädchen (62\% Zustimmung) erklären. Der Blick auf den Migrationshintergrund offenbart dabei sogar noch deutlich stärkere Unterschiede: Drei Viertel (75\%) der Jugendlichen mit Migrationshintergrund gegenüber $56 \%$ ohne einen solchen stimmen traditionellen Werten zu.

23 Traditionelle stimmen Tradition zu und lehnen Hedonismus ab, Hedonisten lehnen Tradition $\mathrm{ab}$ und stimmen Hedonismus zu, Wertesynthetiker stimmen beiden Wertedimensionen zu und die Ablehnenden lehnen beide ab. Als Restkategorie bleiben zwei Typen übrig, die entweder nicht zuordenbar sind oder ein indifferentes Antwortverhalten zeigen.

NK 25. Jg. 3/2013 
lichen bringt damit offenkundig beide Dimensionen in Einklang, was gegen die Inglehartsche These der Unvereinbarkeit von „alten“ und „neuen“ Werten und für die Klagessche Annahme der Wertesynthese spricht.

Bei der Kriminalitätsbelastung der 9. Jahrgangsstufe im Hinblick auf die gesamten Jahresprävalenzen lassen sich in den einzelnen Wertegruppierungen nur geringe Auffälligkeiten feststellen. Bei einer Basisrate von $37 \%$ ist als deutlichster Trend noch die geringere Belastung der Ablehnenden (25\%) auszumachen. Mit insgesamt nur mäßigen und eingeschränkt relevanten Abweichungen lässt sich dennoch festhalten, dass von allen Gruppen die Hedonisten am stärksten belastet sind (43\%), gefolgt von den Wertesynthetikern (42\%). Die Gruppe der Traditionellen hingegen liegt mit $34 \%$ unter der Basisrate. Im Bereich der Sachbeschädigungsdelikte ist einzig die Wertegruppierung der Hedonisten auffällig. Während die Basisrate hier bei $16 \%$ liegt, weisen die Hedonisten eine Täterrate von $23 \%$ auf. Die Wertegruppierung der Ablehnenden ist mit $9 \%$ wiederum deutlich geringer belastet.

Eine Überprüfung nach Geschlecht bringt eine ähnliche Befundlage ohne große Unterschiede in der Gesamtschau hervor. ${ }^{24}$ Die einzige Abweichung zu diesem Gesamtbild ergibt sich im Bereich der Gewaltdelikte, in dem sowohl die männlichen als auch die weiblichen Hedonisten etwas weniger stark belastet sind als der Durchschnitt.

Auch die Analyse des Migrationshintergrundes in Verbindung mit den genannten Wertegruppierungen und der Kriminalitätsbelastung ergibt ein im Großen und Ganzen sehr ähnliches Bild. Von der Grundtendenz sind bei Jugendlichen mit und ohne Migrationshintergrund die Gruppen der Traditionellen und der Ablehnenden geringer und die Gruppen der Hedonisten und Wertesynthetiker stärker belastet. Dies gilt mit der oben schon genannten Einschränkung der geringer belasteten Hedonisten bei den Gewaltdelikten für alle untersuchten Kriminalitätsbereiche. Eine wichtige Erkenntnis ist allerdings, dass sich diese Befundlage deutlich stärker ausgeprägt bei den Jugendlichen ohne Migrationshintergrund präsentiert. Insbesondere bei den Hedonisten ohne Migrationshintergrund im Bereich der Sachbeschädigungsdelikte ( $25 \%$ zu $16 \%$ Basisrate), und den Wertesynthetikern bei den Eigentums- (37\% zu $27 \%$ Basisrate), Gewalt- (19\% zu $11 \%$ Basisrate) und Gesamtprävalenzen ( $53 \%$ zu $37 \%$ Basisrate) sind starke Auffälligkeiten zu berichten. Besonders niedrige Täterraten finden sich in der Gruppe der Ablehnenden ohne Migrationshintergrund bei den Sachbeschädigungsdelikten $(7 \% \mathrm{zu}$ $16 \%$ Basisrate) und den Gesamtprävalenzen ( $21 \%$ zu $37 \%$ Basisrate). Auch wenn die Grundtendenz bei den Jugendlichen mit Migrationshintergrund die gleiche ist, so lassen sich jedoch keine ähnlich starken Auffälligkeiten finden.

24 Sowohl bei den Mädchen, hier jedoch auf einem deutlich niedrigeren Belastungsniveau, als auch bei den Jungen zeigt sich in Bezug auf die Gesamtprävalenzen, die Sachbeschädigungs-, Eigentums- und die Gewaltdelikte eine stärkere Belastung der Hedonisten und der Wertesynthetiker sowie eine geringere Belastung der Traditionellen und der Ablehnenden. Besonders relevant sind dabei die geringeren Belastungen der männlichen Ablehnenden im Bereich der Sachbeschädigungs- (5 \% zu $20 \%$ Basisrate) und der Gewaltdelikte (7 \% zu 19\% Basisrate). 


\section{G. Freizeit und Medien}

\section{Musikgeschmack}

In einer Faktorenanalyse ergeben sich für die 9. Klasse vier Dimensionen des Musikgeschmacks: Harte Musik, Elektro-Musik, ruhige Musik und HipHop/Rap. ${ }^{25}$ Am beliebtesten ist HipHop/Rap (72\%), gefolgt von Elektro-Musik (43\%), ruhiger Musik (15\%) und harter Musik (10\%). Nur HipHop/Rap mag jeder Fünfte (21\%), für Elektro-Musik (6\%), harte Musik (3\%) und ruhige Musik (2\%) gibt es sehr viel weniger, die nur diese Richtung bevorzugen. ${ }^{26}$ Diese Zahlen weisen darauf hin, dass sehr wenige Jugendliche nur bestimmte Musikstile favorisieren.

Während es mehr Jungen gibt, die nur Elektro-Musik mögen (7 \% vs. 5 \%), bevorzugen die Mädchen ruhige Musik stärker als Jungen (4\% vs. $1 \%$ ). Jugendliche ohne Migrationshintergrund bevorzugen häufiger nur harte Musik (5\% vs. $1 \%$ ), Jugendliche mit Migrationshintergrund sind hingegen beim reinen Typ HipHop/Rap häufiger (26\% vs. $15 \%)$. Wie in der Literatur berichtet ${ }^{27}$ sind unter den Jugendlichen, die nur HipHop/ Rap bevorzugen, mehr Gewalttäter als unter denen, die auch andere Stilrichtungen mögen $(18 \%$ vs. $13 \%) .28$

In der 5. Klasse ergeben sich mittels einer Faktorenanalyse drei Musikdimensionen: Elektro-Musik, Schlager-Musik und HipHop/Rap. ${ }^{29}$ Auch hier bevorzugen die meisten HipHop/Rap (70\%), gefolgt von Elektro-Musik (32\%) und Schlager-Musik (17\%). Wie in der 9. Klasse gibt es am meisten Jugendliche, die nur HipHop/Rap mögen (18\%), während sehr wenige nur Elektro-Musik (4\%) oder Schlager-Musik (3\%) bevorzugen. Auch hier scheinen die Jugendlichen gerne Musik eher über verschiedene Richtungen hinweg zu hören. ${ }^{30}$ In der 5 . Klasse ist der Gewalttäteranteil unter den reinen HipHop/Rap-Anhängern jedoch nicht höher als unter denen, die auch andere Musik gerne hören $(1,4 \%$ vs. $1,4 \%)$.

25 Harte Musik: Wave, Gothic; Punk; Hardrock, Metal; Hardcore, Screamo. Elektro-Musik: Techno; Electro; House. Ruhige Musik: Klassik, Oper; Funk, Soul, R'n'B; Blues, Jazz; Country. HipHop/Rap: HipHop; Rap.

26 Die reinen Typen wurden für die 9. (und 5.) Klasse über die Zustimmung zu einer Musikrichtung gebildet, während die anderen drei Musikrichtungen höchstens mittelmäßig gern gehört wurden. Da die Fallzahlen der reinen HipHop/Rap-Richtung zu hoch sind, wurde hier ein strengeres Kriterium angelegt: Zustimmung zu HipHop/Rap bei Ablehnung der anderen drei Richtungen.

27 Pöge 2007.

28 Dies gilt insbesondere für Jugendliche mit Migrationshintergrund (22 \% vs. $14 \%$ ).

29 Elektro-Musik: Techno; Electro. Schlager-Musik: Schlager, Party; Klassik, Oper. HipHop/ Rap: HipHop; Rap.

30 Jungen bevorzugen häufiger reine Elektro-Musik (6 \% vs. $2 \%$ ), Mädchen hingegen häufiger nur Schlager-Musik ( $4 \%$ vs. $2 \%$ ). Jugendliche mit Migrationshintergrund mögen häufiger ausschließlich HipHop/Rap-Musik (36 \% vs. $28 \%$ ).

NK 25. Jg. 3/2013 


\section{Computerspiele}

Eine Faktorenanalyse legt nahe, die Nutzungshäufigkeit der verschiedenen erfragten Computer- und Konsolenspiele in drei Faktoren zusammenzufassen: Sportspiele ${ }^{31}$, Bewegungsspiele ${ }^{32}$ und Rollenspiele ${ }^{33}$. Erwartungskonträr ergibt sich keine Dimension mit rein gewalthaltigen Spielen oder mit dem Item Ego-Shooter/Ballerspiele als definierender Variable. Vielmehr spielen die Jugendlichen anscheinend häufiger Computer- und Konsolenspiele unterschiedlicher Art und weniger gezielt bestimmte Spiele. Auf Einzelitemebene ist sowohl in der 9. Klasse als auch in der 5. Klasse der Täteranteil für diejenigen höher, die Ballerspiele spielen: Der Täteranteil liegt beim häufigen Spielen von EgoShooter/Ballerspielen in der 9. Klasse insgesamt bei $25 \%$ verglichen mit $9 \%$, wenn höchstens manchmal derartige Spiele gespielt werden, für ein Gewaltdelikt bei 52 \% (vs. $30 \%$ ), für ein Sachbeschädigungsdelikt bei $25 \%$ (vs. $13 \%$ ) und ein Eigentumsdelikt bei $39 \%$ (vs. $23 \%$ ). ${ }^{34}$ In der 5 . Klasse ist der Täteranteil ebenso erhöht, wenn Ballerspiele gespielt werden (5. Klasse: Delikte gesamt: $29 \%$ vs. $9 \%$; Gewaltdelikt: $3 \%$ vs. $1 \%$; Sachbeschädigungsdelikt: $21 \%$ vs. $4 \%$; Eigentumsdelikt: $21 \%$ vs. $7 \%$ ). ${ }^{35}$

Die Ergebnisse der Faktorenanalyse in der 9. Klasse weisen darauf hin, dass möglicherweise eher zwischen häufigen und seltenen Spielern insgesamt denn zwischen Spielern von gewalthaltigen und nicht-gewalthaltigen Spielen zu differenzieren ist. So sind die Täteranteile höher, wenn häufig mit dem Computer oder der Konsole gespielt wird, verglichen mit Jugendlichen, die höchstens manchmal spielen (53\% vs. $35 \%)$. Dieser Unterschied in den Täteranteilen gilt auch für Gewaltdelikte ( $28 \%$ vs. $13 \%$ ), Sachbeschädigungsdelikte (26\% vs. $16 \%$ ) und Eigentumsdelikte (39\% vs. $27 \%$ ).

\section{H. Schule, Familie und Peers}

\section{Schule, Schulleistung und Delinquenz}

Die Schule kann grundsätzlich in mehrfacher Hinsicht mit delinquentem Verhalten in Zusammenhang stehen: Als Tatort, als Ort der Bildung (mit Einfluss der Schulleistungen auf Zukunftschancen und Chancen auf soziale Teilhabe) und als soziales System, das über soziale Kontrolle und Vorbilder Delinquenz fördern oder reduzieren kann.

Bezüglich des „Tatorts Schule“ weisen unsere Daten darauf hin, dass die Mehrzahl der Delikte außerhalb der Schule verübt werden, also z.B. im Wohnviertel und in der Innenstadt. Der "Tatort Schule“ wird bei den meisten Delikten in weniger als $20 \%$ der

31 Sportspiele, Sportsimulationen, Rennspiele.

32 Fun-Spiele, Bewegungsspiele, Jump'n'Run.

33 Rollenspiele, Militärstrategiespiele, Adventurespiele, Ego-Shooter/Ballerspiele, Beat'em ups, Aufbauspiele.

34 Differenziert betrachtet sind die Täteranteile unabhängig von Geschlecht oder Migrationshintergrund jeweils höher, wenn Ballerspiele häufig gespielt werden.

35 Das ist unabhängig von Geschlecht oder Migrationshintergrund. Ausnahme hierzu ist, dass bei Jungen die Täteranteile für Gewaltdelikte nicht höher sind, wenn Ballerspiele gespielt werden. 
Fälle genannt. Das einzige Delikt, das überwiegend in der Schule begangen wird, ist das Bestehlen von Mitschülern (5. Klasse $51 \%$; 9 . Klasse $73 \%$ der genannten Tatorte), was schon die Deliktstruktur nahelegt. Als weitere Delikte, die relativ häufig (in über einem Fünftel der Fälle) in der Schule stattfinden, sind „Abziehen“ (Erpressung von Mitschülern), sexuelle Belästigung und Körperverletzung bei Schlägereien zu nennen. ${ }^{36}$ Die Körperverletzung bei einer Schlägerei ist das einzige Delikt, bei dem die Befragten vergleichsweise häufig $(21 \%)$ angeben, ein Lehrer oder die Schulleitung sei darüber informiert. Bei anderen Delikten ist die Kenntnis des Lehrpersonals die Ausnahme (je unter $10 \%)$.

Zur Erfassung der Schulleistungen liegen in unserer Befragung zwei Indikatoren vor: Zum einen der Notendurchschnitt im letzten Zeugnis, zum anderen die Information darüber, ob schon einmal eine Klasse wiederholt wurde. ${ }^{37}$ Schon eine getrennte Betrachtung dieser beiden Indikatoren zeigt, dass Täter häufiger eine Klasse wiederholt haben und einen schlechteren Notendurchschnitt haben als Nichttäter. Diese Unterschiede zeigen sich besonders in der 9. Klasse. ${ }^{38}$ Einen stärkeren Zusammenhang zwischen Schul(miss)erfolg und Delinquenz kann man bei kombinierter Betrachtung beider Indikatoren des Schulerfolgs aufdecken. Da die Wiederholung eines Jahrgangs in der 5. Klasse noch recht selten ist und die Zusammenhänge in der 9. Klasse insgesamt deutlicher ausgeprägt sind, beziehen sich die folgenden Auswertungen nur auf die 9. Jahrgangsstufe. Als Schülerin oder Schüler mit Leistungsproblemen gilt im Folgenden, wer bereits eine Klasse wiederholt hat und im letzten Zeugnis eine Durchschnittsnote schlechter als 3,0 erzielt hat. Durch diese Kombination soll zumindest eine gewisse Schwere und zeitliche Stabilität der Leistungsprobleme indiziert sein. Leistungsprobleme liegen somit in unserer Stichprobe bei 166 Schülerinnen und Schülern der 9. Klasse (12\%) vor. Diese 166 Jugendlichen umfassen Jungen wie Mädchen in ähnlichen Anteilen, der Migrationshintergrund liegt bei $71 \%$. Es zeigt sich, dass der Anteil der Schülerinnen und Schüler mit Leistungsproblemen in allen Deliktklassen und auch bei der Gesamt-Deliktbelastung unter den Tätern deutlich höher ausfällt als unter den Nicht-Tätern (vgl. Tabelle 3). Die Häufung der Leistungsprobleme bei den Tätern findet sich bei Jungen und Mädchen und Teilnehmern mit und ohne Migrationshintergrund in ähnlichem Ausmaß. ${ }^{39}$

36 Die beiden letztgenannten Delikte wurden aufgrund der Deliktschwere nur bei Neuntklässlern erfragt.

37 Rund 38 \% der Fünft- und $41 \%$ der Neuntklässler hatten im letzten Zeugnis einen Notendurchschnitt schlechter als 3,0, $5 \%$ bzw. $3 \%$ sogar einen schlechteren Schnitt als 4,0. Eine Klasse wiederholt haben $9 \%$ der Fünftklässler und $22 \%$ der Neuntklässler.

38 In der 5. Klasse lassen sich die Unterschiede im Notendurchschnitt nur für die Untergruppe der Sachbeschädigungsdelikte finden. Das Wiederholen einer Klasse spielt in der 5. Klasse noch keine Rolle für die Tatfrequenz.

39 Ausnahme: Die Häufung von Schülerinnen und Schülern mit Leistungsproblemen unter den Gewalttätern findet sich nur bei den Befragten mit Migrationshintergrund.

NK 25. Jg. 3/2013 
Tabelle 3: Anteil der Schüler mit Leistungsproblemen unter Tätern und Nichttätern (nur 9. Klasse)

\begin{tabular}{lll}
\hline & \multicolumn{2}{c}{$\%$-Anteil mit Leistungsproblemen } \\
\cline { 2 - 3 } & bei den Nicht-Tätern & bei den Tätern \\
\hline Sachbeschädigung & 15 & 30 \\
Eigentumsdelinquenz & 26 & 38 \\
Gewaltkriminalität & 13 & 24 \\
Delinquenz gesamt & 35 & 51 \\
\hline
\end{tabular}

Dass unzureichende Bildung einen Risikofaktor für spätere Delinquenz darstellt, wurde auch in früheren Untersuchungen belegt ${ }^{40}$. Ein besonders deutlicher Zusammenhang wird in unserer Studie allerdings erst nach Abschluss der Schullaufbahn erwartet, da ein erfolgreicher Schulabschluss als Grundvoraussetzung für eine Berufsausbildung (und somit für soziale Teilhabe) gilt. Die gefundenen Zusammenhänge belegen einerseits die Bedeutung schulischer Bildung für die Delinquenzentwicklung. Andererseits muss natürlich betont werden, dass diese Ergebnisse keinesfalls einseitig kausal interpretiert werden dürfen und die Zusammenhänge insgesamt sehr komplex sind (z.B. Rolle der Schulbindung, Leistungsmotivation, Werte und Normen; frühe Delinquenz als Grund für Ausschluss von „Bildung“, Einfluss eines bildungsfernen oder kriminalitätsbelasteten Elternhauses etc.).

Einfache Häufigkeitsvergleiche, wie hier vorgenommen, können also nur einen ersten Hinweis auf das Bedingungsgefüge delinquenten Verhaltens geben. Die komplexen $\mathrm{Zu}$ sammenhänge zwischen Schule, Elternhaus und Freunden sollen im Folgenden anhand multivariater Analysen näher beleuchtet werden.

\section{Erklärungsfaktoren für Delinquenz}

Legt man eine kontrolltheoretische Perspektive ${ }^{41}$ zugrunde, kann man davon ausgehen, dass eine positive Bindung an Eltern und Schule (wie beispielsweise eine gute Kommunikation in der Familie oder ein gutes Vertrauensverhältnis zu Mitschülern oder Lehrern) einen Delinquenz reduzierenden Einfluss ausübt. Gewalttätige, inkonsistente sowie vernachlässigende Erziehungsstile steigern hingegen die Wahrscheinlichkeit delinquenten Verhaltens. Dabei können die Einflüsse direkt, indirekt oder vermittelt über delinquente Peers ihre Wirkung entfalten. Die Annahme dahinter ist, dass Kinder und Jugendliche mit starken sozialen Bindungen (zu Eltern und/oder Schule) weniger geneigt sind, sich einer delinquenten Peergruppe anzuschließen. Zudem kann aus lerntheoretischer Per- 
spektive $^{42}$ davon ausgegangen werden, dass delinquente Peers bspw. wenn deren Verhalten imitiert wird, einen direkten Einfluss auf das eigene delinquente Verhalten ausüben.

Betrachtet man den Einfluss von Familie, Schule und Peers auf die selbstberichtete Delinquenz in multivariaten Analysen ${ }^{43}$, ergeben sich in den unterschiedlichen Klassenstufen divergierende Ergebnisse. Ob ein Jugendlicher der 5. Jahrgangsstufe zum Täter wird, ist dabei direkt sowie zusätzlich vermittelt über einen delinquenten Freundeskreis, beeinflusst durch eine schwache Bindung an die Schule und durch inkonsistente sowie vernachlässigende Erziehungsstile der Eltern (vgl. Schaubild 2). Ein indirekter, die selbstberichtete Delinquenz reduzierender Effekt zeigt sich für eine positive Bindung an die Eltern (gemessen durch die Dimension Vertrauen). Indirekte, die Delinquenz steigernde Effekte zeigen sich hingegen für die Bindungsdimension Kommunikation und eine gewalttätige Erziehung. ${ }^{44}$

Schaubild 2: SEM-Modell der 5. Klasse

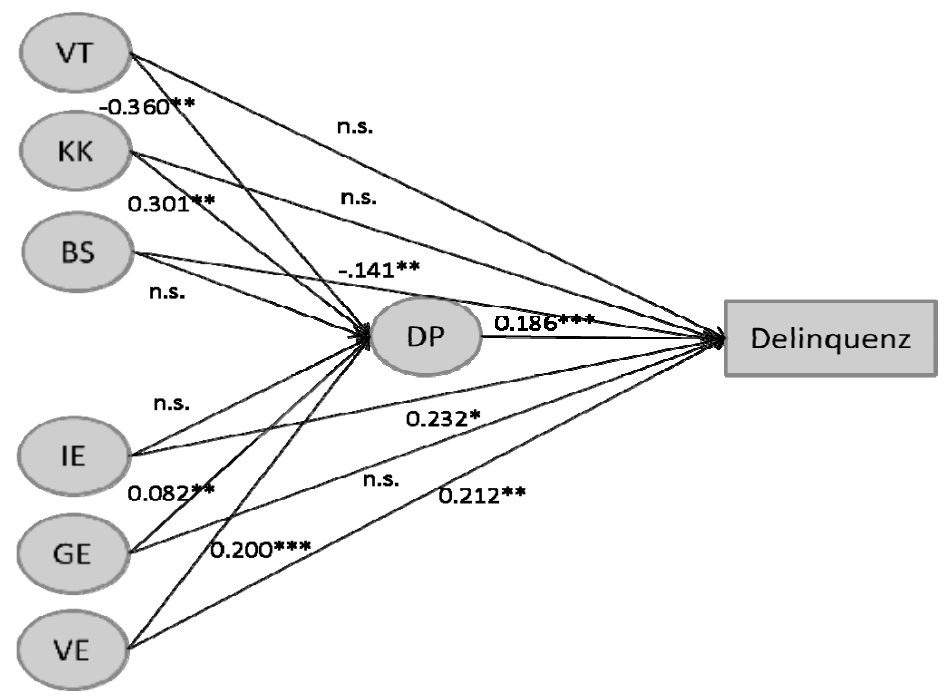

Anmerkungen: Standardisierte Ergebnisse; $\mathrm{N}=1327 ; \chi^{2}=515.762, \mathrm{df}=182 ; \mathrm{RMSEA}=0.037$; $\mathrm{CFI}=0.954$; Signifikanzniveau: $* * \mathrm{*}<0.001$, $* \mathrm{p}<0.01, * \mathrm{p}<0.05 ;$ n.s. $=$ nicht signifikant; Bedeutung der Regressionskoeffizienten: bis 0.14 - sehr schwacher Zusammenhang; 0.15 bis 0.19 - schwach; 0.20 bis 0.29 - moderat; 0.30 bis 0.39 - stärker; 0.40 bis 0.49 - stark; $\mathrm{VT}=$ Vertrauen, $\mathrm{KK}=\mathrm{Kom}$ munikation, $\mathrm{BS}=$ Bindung an die Schule, $\mathrm{IE}=$ inkonsistenter Erziehungsstil, $\mathrm{GE}=$ gewalttätiger Erziehungsstil, VE= vernachlässigender Erziehungsstil, DP= delinquente Peers.

42 Vgl. Sutherland/Cressey 1960.

43 Für die multivariaten Analysen wurden Strukturgleichungsmodelle berechnet (vgl. Reinecke 2005). Indirekte und mediierende Effekte werden nicht gesondert ausgewiesen.

44 Erklärungsbedürftig ist hierbei der positive Effekt der Kommunikation, welcher in folgenden Analysen noch genauer untersucht werden sollte.

NK 25. Jg. 3/2013 
In der 9. Jahrgangsstufe finden sich nur für vernachlässigende Erziehungsstile und delinquente Peers direkte steigernde Effekte auf das delinquente Verhalten (vgl. Schaubild 3). Gewalttätige sowie vernachlässigende Erziehungsstile und eine geringe Bindung an die Schule haben zudem einen direkten Einfluss auf die Wahrscheinlichkeit, delinquente Freunde zu haben. In der 9. Klasse wirken sich indirekt ebenfalls gewalttätige und vernachlässigende Erziehungsstile sowie die Bindung an die Schule auf die Delinquenz aus.

\section{Schaubild 3: SEM-Modell der 9. Klasse}

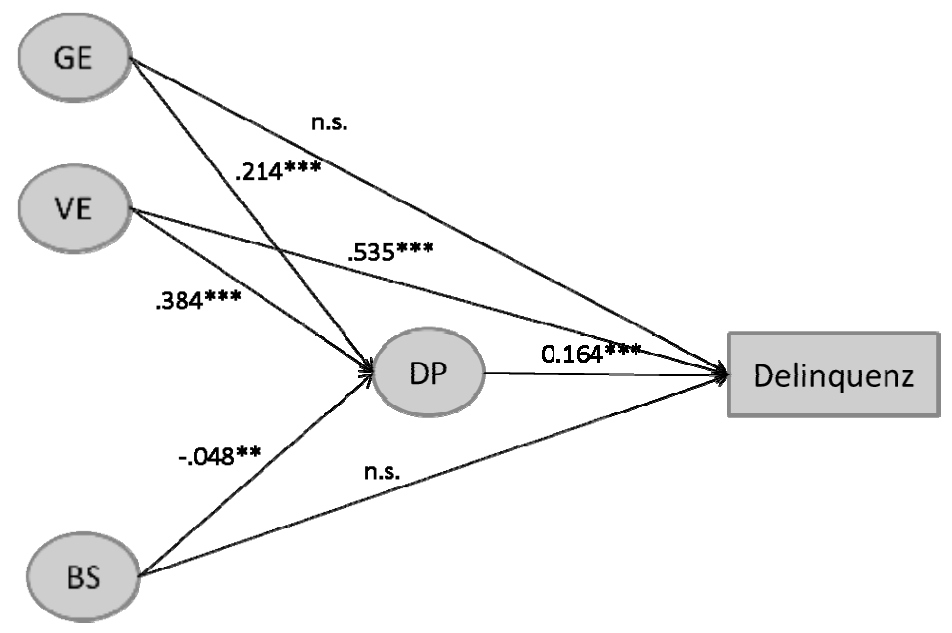

Anmerkungen: Standardisierte Ergebnisse; $\mathrm{N}=1416 ; \quad \mathrm{X}^{2}=202.778, \mathrm{df}=68 ; \quad \mathrm{RMSEA}=0.037$; CFI=0.970; Signifikanzniveau: $* * * \mathrm{p}<0.001, * * \mathrm{p}<0.01,{ }^{*} \mathrm{p}<0.05$; n.s. $=$ nicht signifikant; Bedeutung der Regressionskoeffizienten: bis 0.14 - sehr schwacher Zusammenhang; 0.15 bis 0.19 - schwach; 0.20 bis 0.29 - moderat; 0.30 bis 0.39 - stärker; 0.40 bis 0.49 - stark; $\mathrm{GE}=$ gewalttätiger Erziehungsstil, $\mathrm{VE}=$ vernachlässigender Erziehungsstil, $\mathrm{BS}=$ Bindung an die Schule, $\mathrm{DP}=$ delinquente Peers.

Bei beiden Jahrgängen wirkt eine vernachlässigende Erziehung direkt, indirekt und mediiert über delinquente Peers auf die selbstberichtete Delinquenz. Die Ergebnisse können damit für beide Jahrgänge die These stützen, dass ein vernachlässigender Erziehungsstil (Abwesenheit sozialer Kontrolle-schwache Bindung) dazu führt, sich eher delinquenten Peers anzuschließen, und somit eigenes delinquentes Verhalten wahrscheinlicher macht. ${ }^{45}$ Inkonsistente Erziehungsstile und die Bindung an Eltern und Schule verlieren in der 9. Jahrgangsstufe hingegen an Wert für die Erklärung delinquenten Verhaltens.

45 Gegner der Selektionsthese würden behaupten, dass sich die Jugendlichen eher aufgrund von Ähnlichkeiten in der Persönlichkeitsstruktur oder anderen Gründen zusammentun, bzw. aufgrund vorherigen delinquenten Verhaltens eine Stigmatisierung stattgefunden haben könnte, was eine Teilnahme an einer nicht-delinquenten Gruppe unwahrscheinlicher werden lässt. Um feststellen zu können, welcher der Standpunkte sich empirisch bestätigt, sind allerdings Längsschnittuntersuchungen erforderlich. 
Diese Ergebnisse stützen damit die Annahmen der „Age Graded Theory“46, wonach Einflüsse der Schule und der Familie im Heranwachsendenalter abnehmen.

\section{Ausblick}

Die bisher vorliegenden Ergebnisse beziehen sich auf die ersten Erhebungen in den Städten Dortmund und Nürnberg. Drei weitere Wiederholungsuntersuchungen mit den gleichen Personen sind für die Jahre 2013 (derzeit in der Feldphase), 2014 und 2015 geplant. Das Analysedesign ermöglicht die Erhebung eines Datenumfangs vom Jugendalter bis in das junge Erwachsenenalter, um sowohl indirekte als auch direkte Zusammenhänge von Persönlichkeitsmerkmalen und sozialstrukturellen Größen mit unterschiedlichen Verlaufspfaden der Devianz und Delinquenz zu analysieren. Damit wird auch das bisher vorliegende Defizit an kriminologischen Verlaufsuntersuchungen in Deutschland geringer.

\section{Literatur}

Baier, D. \& Pfeiffer, C. (2011), Jugendliche als Opfer und Täter von Gewalt in Berlin, Forschungsbericht Nr. 120, Hannover: KFN

Baier, D., Pfeiffer, C., Rabold, S., Simonson, J. \& Kappes, C. (2010a), Kinder und Jugendliche in Deutschland: Gewalterfahrungen, Integration, Medienkonsum. Zweiter Bericht zum gemeinsamen Forschungsprojekt des Bundesministeriums des Innern und des KFN, Forschungsbericht Nr. 109 Hannover: KFN

Baier, D., Pfeiffer, C., Simonson, J. \& Rabold, S. (2009), Jugendliche in Deutschland als Opfer und Täter von Gewalt. Erster Forschungsbericht zum gemeinsamen Forschungsprojekt des Bundesministeriums des Innern und des KFN Forschungsbericht Nr. 107 Hannover: KFN

Baier, D., Pfeiffer, C. \& Windzio, M. (2006a), Jugendliche mit Migrationshintergrund als Opfer und Täter. Fachwissenschaftliche Analyse, in: Heitmeyer \& Schröttle (Hrsg.), Gewalt. Beschreibungen, Analysen, Prävention (240-275), Bonn

Baier, D., Pfeiffer, C., Windzio, M. \& Rabold, S. (2006b), Schülerbefragung 2005: Gewalterfahrungen, Schulabsentismus und Medienkonsum von Kindern und Jugendlichen. Abschlussbericht über eine repräsentative Befragung von Schülerinnen und Schülern der 4. und 9. Jahrgangsstufe, Hannover: KFN 
Baier, D. \& Rabold, S. (2012), Kinder- und Jugenddelinquenz im Bundesland Saarland, Forschungsbericht Nr. 120, Hannover: KFN

Baier, D., Rabold, S. \& Doering, B. (2010b), Jugendliche als Opfer und Täter von Gewalt im Bundesland Sachsen-Anhalt, Forschungsbericht Nr. 110, Hannover: KFN

Bayerisches Landeskriminalamt (Hrsg.) (2001-2013), Jahrbuch Polizeiliche Kriminalstatistik für den Freistaat Bayern, München: Landeskriminalamt

Bliesener, T., Beelmann, A. \& Stemmler, M. (Eds.) (2012), Antisocial Behavior \& Crime, Göttingen: Hogrefe

Boers, K. \& Pöge, A. (2002), Wertorientierungen und Jugenddelinquenz, in: Lamnek \& Boatca (Hrsg.), Geschlecht, Gewalt, Gesellschaft (246-269), Opladen: Leske + Budrich

Bundeskriminalamt (Hrsg.) (2001-2013), Polizeiliche Kriminalstatistik der Bundesrepublik Deutschland, Wiesbaden: Bundeskriminalamt

Bundeskriminalamt (Hrsg.) (2013),Polizeiliche Kriminalstatistik 2012 - Zeitreihen Tatverdächtige. Tabellen 20, 40 und 50. www.bka.de

Bundeszentrale für gesundheitliche Aufklärung ( $B Z g A)$ (2012), Die Drogenaffinität Jugendlicher in der Bundesrepublik Deutschland 2011. Der Konsum von Alkohol, Tabak und illegalen Drogen: Aktuelle Verbreitung und Trends, Köln: Bundeszentrale für gesundheitliche Aufklärung

Entorf, H. \& Sieger, P. (2010), Unzureichende Bildung: Folgekosten durch Kriminalität, Gütersloh: Bertelsmann Stiftung

ESPAD (European school survey on alcohol and other drugs) (2011), The 2011 ESPADReport, http://www.espad.org/Uploads/ESPAD_reports/2011/The_2011_ESPAD_Report_SUMMARY.pdf, Abruf am 28.05.2013

Flaig, B. B., Meyer, T. \& Ueltzhöffer, J. (1994), Alltagswelt, Lebensstile und soziale Milieus, in: Flaig, Meyer \& Ueltzhöffer (Hrsg.), Alltagsästhetik und politische Kultur. Zur ästhetischen Dimension politischer Bildung und politischer Kommunikation (33-74), 2. Aufl., Bonn: J. H.W. Dietz Nachf. GmbH

Gottfredson, M. R. \& Hirschi, T. (1990), A general theory of crime, Stanford: Calif. Stanford Univ. Press 
Heitmeyer, W., Collmann, B., Conrads, J., Matuschek, I., Kraul, D., Kühnel, W. et al. (1995), Gewalt. Schattenseiten der Individualisierung bei Jugendlichen aus unterschiedlichen Milieus, Weinheim und München: Juventa

Hermann, D. (2003), Werte und Kriminalität, Konzeption einer allgemeinen Kriminalitätstheorie, Wiesbaden: Westdeutscher Verlag

Hirschi, T. (1969), Causes of Delinquency, Berkley: University of California Press

Hurrelmann, K. (1997), Lebensphase Jugend. Eine Einführung in die sozialwissenschaftliche Jugendforschung, Weinheim und München: Juventa

Inglehart, R. (1979), Wertewandel in den westlichen Gesellschaften: Politische Konsequenzen von materialistischen und postmaterialistischen Prioritäten, in: Klages \& Kmieciak (Hrsg.), Wertewandel und gesellschaftlicher Wandel (279-317), Frankfurt a. M.: Campus

Klages, H. (1984), Wertorientierungen im Wandel. Rückblick, Gegenwartsanalysen, Prognosen, Frankfurt a. M.: Campus

Klages, H. \& Gensicke, T. (2006), Wertesynthese - funktional oder dysfunktional?, in: Kölner Zeitschrift für Soziologie und Sozialpsychologie, 58 (2), 332-351

Landeskriminalamt Nordrhein-Westfalen (Hrsg.) (2001-2013) Polizeiliche Kriminalstatistik. Kriminalitätsentwicklung in NRW, Düsseldorf: Landeskriminalamt

Landeskriminalamt Nordrbein-Westfalen (2013) PKS Stadt Dortmund, persönliche Mitteilung der Kriminologischen Forschungsstelle des Landeskriminalamtes, Düsseldorf

Pöge, A. (2007), Soziale Milieus und Delinquenz im Jugendalter, Münster: Waxmann

Polizeipräsidium Mittelfranken (Hrsg.) (2013), Kriminalstatistik 2012, Nürnberg: Polizeipräsidium

Reinecke, J. (2005), Strukturgleichungsmodelle in den Sozialwissenschaften, München: Oldenbourg Verlag

Reinecke, J. (2007), Das Verhältnis von Wertorientierungen, Freizeitstilen, Rechtsnormen und Delinquenz, in: Boers \& Reinecke (Hrsg.), Delinquenz im Jugendalter. Erkenntnisse einer Münsteraner Längsschnittstudie (335-358). Münster u. a.: Waxmann

Reinecke, J. (2012), Wachstumsmodelle. Sozialwissenschaftliche Forschungsmethoden, Band 3, München: Rainer Hampp Verlag

NK 25. Jg. 3/2013 
Reinecke, J., Stemmler, M., Sünkel, Z., Schepers, D., Weiss, M., Arnis, M., Meinert, J., Kucur-Uysal, B., Pöge, A., Wallner, S. \& Wittenberg, J. (2013), The development of deviant and delinquent behavior over the life course in the context of processes of social inequalities, SFB 882 Working Paper Series, no. 17, Bielefeld: DFG Research Center (SFB) 882 From Heterogeneities to Inequalities

Sampson, R. J. \& Laub, J. H. (1993), Crime in the Making: Pathways and Turning Points through Life, Cambridge, MA: Harvard University Press

Statistisches Bundesamt (2012), Bevölkerung und Erwerbstätigkeit. Bevölkerung mit Migrationshintergrund - Ergebnisse des Mikrozensus 2011, Wiesbaden https:// www.destatis.de/DE/Publikationen/Thematisch/Bevoelkerung/MigrationIntegration/ Migrationshintergrund 2010220117004.pdf?_blob=publicationFile, Abruf am 13.06.2013

Strohmeier, D. (2007), Soziale Beziehungen in multikulturellen Schulklassen: Wo liegen die Chancen, wo die Risiken?, in: Erziehung und Unterricht, November/Dezember 9-10/2007, 796-807

Sutberland, E. \& Cressey, D. (1960), Principles of Criminology. Philadelphia: Lippincott

Wittenberg, J., Reinecke, J. \& Boers, K. (2011), Verbreitung und Verläufe von Jugenddelinquenz: Befunde und Erklärungsansätze im Rahmen von Längsschnittstudien in Münster und Duisburg, in: Tarnai (Hrsg.), Sozialwissenschaftliche Forschung in Diskurs und Empirie (119-140), Münster: Waxmann

Jost Reinecke, Nibad El-Kayed, Julia Meinert, Andreas Pöge, Deborah Schepers, Jochen Wittenberg

Universität Bielefeld

Fakultät für Soziologie

Universitätsstraße 25

33615 Bielefeld

Mark Stemmler, Maria Arnis, Zara Sünkel, Burcu Uysal, Susanne Wallner, Maren Weiss Universität Erlangen-Nürnberg

Philosophische Fakultät und Fachbereich Theologie

Nägelsbachstraße 49c

91052 Erlangen 